\title{
Anomalous dimensions and splitting functions beyond the next-to-next-to-leading order
}

\section{A. Vogt*}

Department of Mathematical Sciences, University of Liverpool, Liverpool L69 3BX, UK E-mail: Andreas.Vogteliverpool.ac.uk

\section{F. Herzog}

Nikhef Theory Group, Science Park 105, 1098 XG Amsterdam, The Netherlands E-mail: fherzogenikhef.nl

\section{S. Moch}

II. Institut für Theoretische Physik, Universität Hamburg, D-22761 Hamburg, Germany E-mail: sven-olaf.moch@desy.de

\section{B. Ruijl}

Institute for Theoretical Physics, ETH Zürich, 8093 Zürich, Switzerland E-mail: bruijlephys.ethz.ch

\section{T. Ueda}

Department of Materials and Life Science, Seikei University, Musashino, Tokyo 180-8633, Japan E-mail: tuedalst.seikei.ac.jp

\section{J.A.M. Vermaseren}

Nikhef Theory Group, Science Park 105, 1098 XG Amsterdam, The Netherlands

E-mail: t68@nikhef.nl

\begin{abstract}
We report on recent progress on the splitting functions for the evolution of parton distributions and related quantities, the (lightlike) cusp anomalous dimensions, in perturbative QCD. New results are presented for the four-loop (next-to-next-to-next-to-leading order, $\mathrm{N}^{3} \mathrm{LO}$ ) contributions to the flavour-singlet splitting functions and the gluon cusp anomalous dimension. We present first results, the moments $N=2$ and $N=3$, for the five-loop $\left(\mathrm{N}^{4} \mathrm{LO}\right)$ non-singlet splitting functions.
\end{abstract}

Loops and Legs in Quantum Field Theory (LL2018)

29 April 2018 - 04 May 2018, St. Goar, Germany

\footnotetext{
* Speaker.
} 


\section{Introduction}

Up to power corrections, observables in $e p$ and $p p$ hard scattering can be schematically expressed as

$$
O^{e p}=f_{i} \otimes c_{i}^{\mathrm{o}}, \quad O^{p p}=f_{i} \otimes f_{k} \otimes c_{i k}^{\mathrm{o}}
$$

in terms of the respective partonic cross sections (coefficient functions) $c^{\mathrm{o}}$ and the universal parton distribution functions (PDFs) $f_{i}\left(x, \mu^{2}\right)$ of the proton at a scale $\mu$ of the order of a physical scale. The dependence of the PDFs on the momentum fraction $x$ is not calculable in perturbative QCD; their scale dependence is given by the renormalization-group evolution equations

$$
\frac{\partial}{\partial \ln \mu^{2}} f_{i}\left(x, \mu^{2}\right)=\int_{x}^{1} \frac{d y}{y} P_{i k}\left(y, \alpha_{\mathrm{s}}\left(\mu^{2}\right)\right) f_{k}\left(\frac{x}{y}, \mu^{2}\right) .
$$

The splitting functions $P_{i k}$, which are closely related to the anomalous dimensions of twist-2 operators in the light-cone operator-product expansion (OPE), and the coefficient functions in eq. (1.1) can be expanded in powers of the strong coupling $a_{\mathbf{s}} \equiv \alpha_{\mathbf{s}}\left(\mu^{2}\right) /(4 \pi)$,

$$
\begin{gathered}
P=a_{\mathrm{s}} P^{(0)}+a_{\mathrm{s}}^{2} P^{(1)}+a_{\mathrm{s}}^{3} P^{(2)}+a_{\mathrm{s}}^{4} P^{(3)}+\ldots, \\
c_{a}^{\mathrm{o}}=a_{\mathrm{s}}^{n_{\mathrm{o}}}\left[c_{\mathrm{o}}^{(0)}+a_{\mathrm{s}} c_{\mathrm{o}}^{(1)}+a_{\mathrm{s}}^{2} c_{\mathrm{o}}^{(2)}+a_{\mathrm{s}}^{3} c_{\mathrm{o}}^{(3)}+\ldots\right] .
\end{gathered}
$$

Together, the first three terms of eqs. (1.3) and (1.4) provide the NNLO approximation for the observables (1.1). This is now the standard accuracy of perturbative QCD for many hard processes; see refs. [1,2] for the corresponding helicity-averaged and helicity-dependent splitting functions. $\mathrm{N}^{3} \mathrm{LO}$ corrections have been obtained for inclusive lepton-hadron deep-inelastic scattering (DIS) [3], Higgs production in proton-proton collisions [4,5], and jet production in DIS [6]. $\mathrm{N}^{4} \mathrm{LO}$ results for inclusive DIS have been reported in refs. [7] (sum rules) and ref. [8] (low Mellin- $N$ moments).

Using basic symmetries, the system (1.2) can be decomposed into $2 n_{f}-1$ scalar 'non-singlet' equations and a $2 \times 2$ flavour-singlet system. The former includes $2\left(n_{f}-1\right)$ flavour asymmetries of quark-antiquark sums and differences, $q_{i} \pm \bar{q}_{i}$, and the total valence distribution,

$$
q_{\mathrm{ns}, i k}^{ \pm}=q_{i} \pm \bar{q}_{i}-\left(q_{k} \pm \bar{q}_{k}\right), \quad q_{\mathrm{v}}=\sum_{r=1}^{n_{f}}\left(q_{r}-\bar{q}_{r}\right) .
$$

The singlet PDFs and their evolution are given by

$$
q_{\mathrm{s}}=\sum_{r=1}^{n_{f}}\left(q_{r}+\bar{q}_{r}\right), \quad \frac{d}{d \ln \mu^{2}}\left(\begin{array}{c}
q_{\mathrm{s}} \\
g
\end{array}\right)=\left(\begin{array}{c}
P_{\mathrm{qq}} P_{\mathrm{qg}} \\
P_{\mathrm{gq}}
\end{array} P_{\mathrm{gg}}\right) \otimes\left(\begin{array}{c}
q_{\mathrm{s}} \\
g
\end{array}\right)
$$

where $g\left(x, \mu^{2}\right)$ denotes the gluon distribution. $P_{\mathrm{qq}}$ differs from the splitting function $P_{\mathrm{ns}}^{+}$for the combinations $q_{\mathrm{ns}, i k}^{+}$in Eq. (1.5) by a pure singlet contribution $P_{\mathrm{ps}}$ which is suppressed at large $x$. In this limit, the splitting functions $P_{\mathrm{qq}}$ and $P_{\mathrm{gg}}$ in the standard $\overline{\mathrm{MS}}$ scheme are of the form

$$
P_{\mathrm{kk}}^{(n-1)}(x)=\frac{x A_{n, \mathrm{k}}}{(1-x)_{+}}+B_{n, \mathrm{k}} \delta(1-x)+C_{n, \mathrm{k}} \ln (1-x)+D_{n, \mathrm{k}}+(1-x) \text {-terms },
$$

where $A_{n, \mathrm{q}}$ and $A_{n, \mathrm{~g}}$ are the (light-like) $n$-loop quark and gluon cusp anomalous dimensions [9]. These and the 'virtual anomalous dimensions' $B_{n, \mathrm{k}}$ are relevant well beyond the context of Eq. (1.2).

In this contribution we briefly report on recent $\mathrm{N}^{3} \mathrm{LO}$ (4-loop) results for the singlet splitting functions in eq. (1.6), including the gluon cusp anomalous dimension $A_{4, \mathrm{~g}}$ [10], and on the first $\mathrm{N}^{4} \mathrm{LO}$ (5-loop) calculations of the non-singlet splitting functions $P_{\mathrm{ns}}^{ \pm}$. For the (more advanced) status of the 4-loop non-singlet splitting functions the reader is referred to refs. [11-13]. 


\section{Low- $N$ results for the $\mathbf{N}^{3} \mathrm{LO}$ singlet splitting functions}

The results for $N=2$ and $N=4$ have been reported, in numerical form for $n_{f}=4$ flavours in QCD, at the previous Loops \& Legs Workshop [8]. In the meantime, the computations of four-loop DIS with FORCER [14], which are conceptually straightforward extensions of the three-loop calculations in refs. [15], have been extended to $N=6$ for $P_{\mathrm{qg}}^{(3)}$ and $P_{\mathrm{gg}}^{(3)}$ and to $N=8$ for $P_{\mathrm{qq}}^{(3)}$ and $P_{\mathrm{gq}}^{(3)}$. The resulting perturbative expansions of $P_{i k}\left(N, n_{f}=4\right)$ are approximately given by

$$
\begin{aligned}
& P_{\mathrm{qq}}(2,4)=-0.28294 \alpha_{\mathrm{s}}\left(1+0.6219 \alpha_{\mathrm{s}}+0.1461 \alpha_{\mathrm{s}}^{2}+0.3622 \alpha_{\mathrm{s}}^{3}+\ldots\right), \\
& P_{\mathrm{qq}}(4,4)=-0.55527 \alpha_{\mathrm{s}}\left(1+0.6803 \alpha_{\mathrm{s}}+0.4278 \alpha_{\mathrm{s}}^{2}+0.3459 \alpha_{\mathrm{s}}^{3}+\ldots\right), \\
& P_{\mathrm{qq}}(6,4)=-0.71645 \alpha_{\mathrm{s}}\left(1+0.6489 \alpha_{\mathrm{s}}+0.4264 \alpha_{\mathrm{s}}^{2}+0.3248 \alpha_{\mathrm{s}}^{3}+\ldots\right), \\
& P_{\mathrm{qq}}(8,4)=-0.83224 \alpha_{\mathrm{s}}\left(1+0.6328 \alpha_{\mathrm{s}}+0.4235 \alpha_{\mathrm{s}}^{2}+0.3121 \alpha_{\mathrm{s}}^{3}+\ldots\right), \\
& P_{\mathrm{qg}}(2,4)=0.21221 \alpha_{\mathrm{s}}\left(1+0.9004 \alpha_{\mathrm{s}}-0.1028 \alpha_{\mathrm{s}}^{2}-0.2367 \alpha_{\mathrm{s}}^{3}+\ldots\right), \\
& P_{\mathrm{qg}}(4,4)=0.11671 \alpha_{\mathrm{s}}\left(1-0.2801 \alpha_{\mathrm{s}}-0.9986 \alpha_{\mathrm{s}}^{2}+0.1297 \alpha_{\mathrm{s}}^{3}+\ldots\right), \\
& P_{\mathrm{qg}}(6,4)=0.08337 \alpha_{\mathrm{s}}\left(1-0.8389 \alpha_{\mathrm{s}}-1.1501 \alpha_{\mathrm{s}}^{2}+0.4417 \alpha_{\mathrm{s}}^{3}+\ldots\right), \\
& P_{\mathrm{gq}}(2,4)=0.28294 \alpha_{\mathrm{s}}\left(1+0.6219 \alpha_{\mathrm{s}}+0.1461 \alpha_{\mathrm{s}}^{2}+0.3622 \alpha_{\mathrm{s}}^{3}+\ldots\right), \\
& P_{\mathrm{gq}}(4,4)=0.07781 \alpha_{\mathrm{s}}\left(1+1.1152 \alpha_{\mathrm{s}}+0.8234 \alpha_{\mathrm{s}}^{2}+0.8833 \alpha_{\mathrm{s}}^{3}+\ldots\right), \\
& P_{\mathrm{gq}}(6,4)=0.04446 \alpha_{\mathrm{s}}\left(1+1.3019 \alpha_{\mathrm{s}}+1.0516 \alpha_{\mathrm{s}}^{2}+1.1270 \alpha_{\mathrm{s}}^{3}+\ldots\right), \\
& P_{\mathrm{gq}}(8,4)=0.03116 \alpha_{\mathrm{s}}\left(1+1.4309 \alpha_{\mathrm{s}}+1.1830 \alpha_{\mathrm{s}}^{2}+1.3184 \alpha_{\mathrm{s}}^{3}+\ldots\right)
\end{aligned}
$$

and

$$
\begin{aligned}
& P_{\mathrm{gg}}(2,4)=-0.21221 \alpha_{\mathrm{s}}\left(1+0.9004 \alpha_{\mathrm{s}}-0.1028 \alpha_{\mathrm{s}}^{2}-0.2367 \alpha_{\mathrm{s}}^{3}+\ldots\right) \\
& P_{\mathrm{gg}}(4,4)=-1.21489 \alpha_{\mathrm{s}}\left(1+0.3835 \alpha_{\mathrm{s}}+0.1220 \alpha_{\mathrm{s}}^{2}+0.2405 \alpha_{\mathrm{s}}^{3}+\ldots\right) \\
& P_{\mathrm{gg}}(6,4)=-1.62755 \alpha_{\mathrm{s}}\left(1+0.3937 \alpha_{\mathrm{s}}+0.1697 \alpha_{\mathrm{s}}^{2}+0.1902 \alpha_{\mathrm{s}}^{3}+\ldots\right)
\end{aligned}
$$

The corresponding analytic expressions for a general gauge group will be presented elsewhere.

The relative size of the $\mathrm{N}^{2} \mathrm{LO}$ and $\mathrm{N}^{3} \mathrm{LO}$ contributions to eqs. 2.1. -2.4 is illustrated in fig. 1 for $\alpha_{\mathrm{s}}=0.2$ : The $\mathrm{N}^{3} \mathrm{LO}$ corrections are less than $1 \%$, and less than $0.5 \%$ of the NLO results except for $P_{\mathrm{gq}}$, the quantity with the lowest LO values, at $N \geq 4$.

The resulting low- $N$ expansion for the singlet evolutions equations (1.6) is illustrated in fig. 2 for the sufficiently realistic order-independent model input

$$
\begin{gathered}
x q_{\mathrm{s}}\left(x, \mu_{0}^{2}\right)=0.6 x^{-0.3}(1-x)^{3.5}\left(1+5.0 x^{0.8}\right), \\
x g\left(x, \mu_{0}^{2}\right)=1.6 x^{-0.3}(1-x)^{4.5}\left(1-0.6 x^{0.3}\right)
\end{gathered}
$$

with $\alpha_{\mathrm{s}}\left(\mu_{0}^{2}\right)=0.2$ and $n_{f}=4$, which was already used in ref. [1]. The $\mathrm{N}^{3} \mathrm{LO}$ corrections are very small at the standard renormalization scale $\mu_{r}=\mu_{f} \equiv \mu_{0}$. They lead to a reduction of the scale dependence to about $1 \%$ (full width) at $N=4 \& N=6$ for the conventional range $\frac{1}{4} \mu_{f}^{2} \leq \mu_{r}^{2} \leq 4 \mu_{f}^{2}$.

Extending eqs. (2.2) and (2.4) to $N=8$ would be extremely hard with the hardware and software used to obtain these results; computing the $N=10$ results in this way is virtually impossible. 

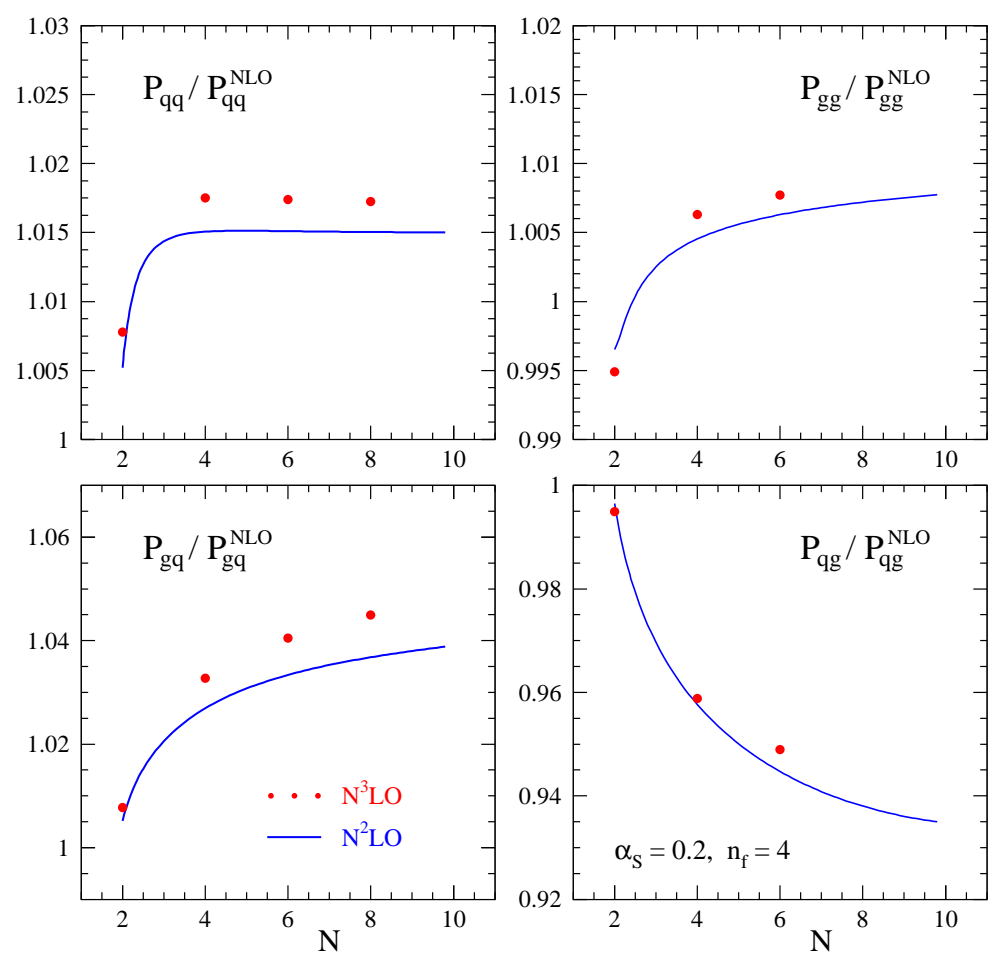

Figure 1: Moments of the singlet splitting functions at NNLO (lines) and $\mathrm{N}^{3} \mathrm{LO}$ (even- $N$ points) for $\alpha_{\mathrm{s}}=0.2$ and $n_{f}=4$, normalized to the respective NLO approximations.
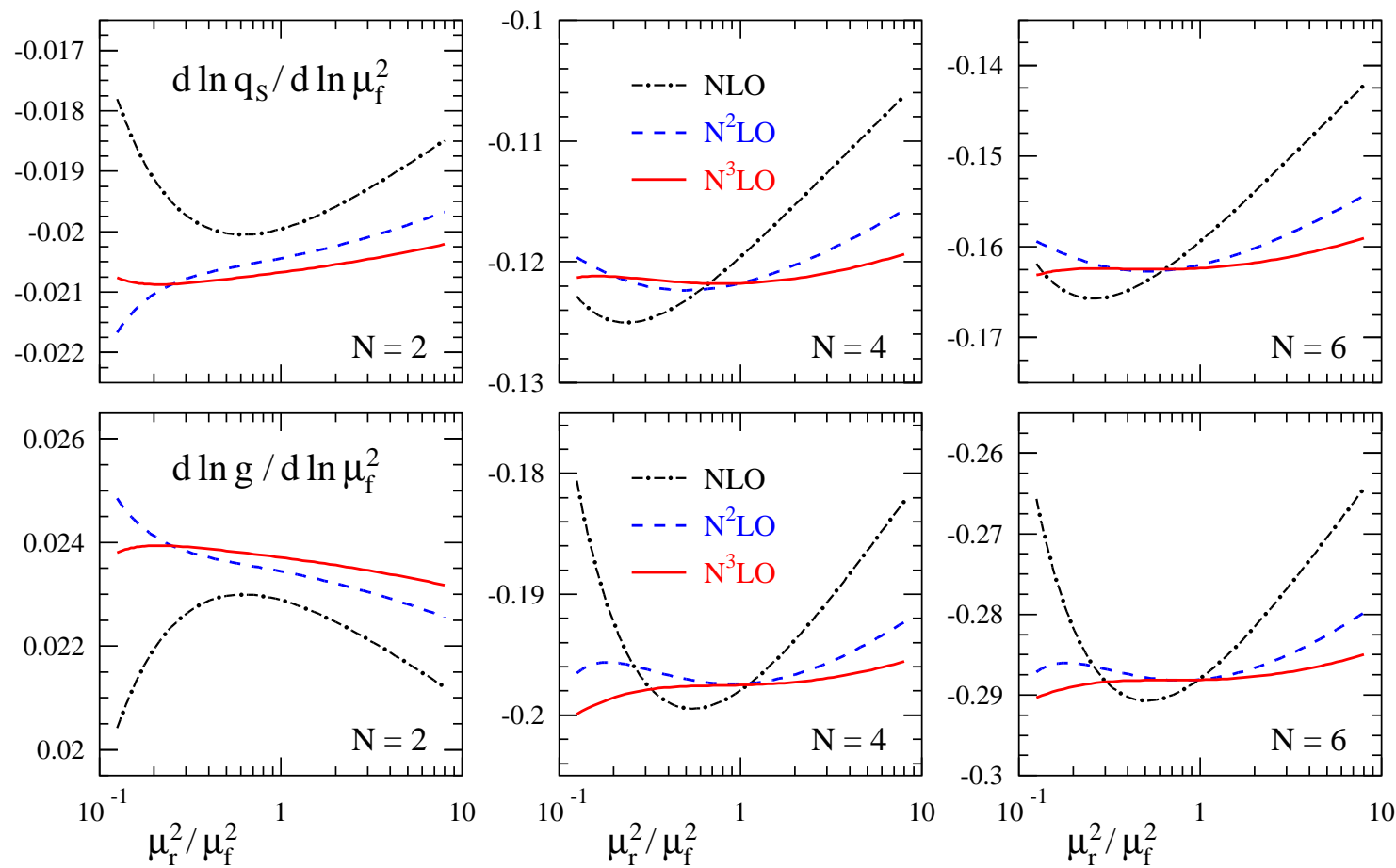

Figure 2: The dependence of the logarithmic factorization-scale derivatives of the singlet PDFs on the renormalization scale $\mu_{r}$ at $N=2$ (where the very small scaling violations of $q_{\mathrm{s}}$ and $g$ are related by the momentum sum rule) $N=4$ and $N=6$ for the initial distributions (2.5). 


\section{Quartic colour-factor contributions and the cusp anomalous dimensions}

The computations of the four-loop splitting functions can be extended to higher $N$ by using the OPE, since there the complexity of the required self-energy integral increases by 2 for $N \rightarrow N+2$ instead of by 4 in the case of DIS. For example, $N=16$ has been reached for the complete $\mathrm{N}^{3} \mathrm{LO}$ contribution to $P_{\mathrm{ns}}^{+}$. In the limit of a large number of colours $n_{c}$, it was possible to reach $N=20$, which led to the determination of the all- $N$ expressions and hence of $P_{\mathrm{ns}}^{ \pm(3)}(x)$ in this limit [12].

In general the higher-order application of the OPE in massless perturbative QCD is conceptually much more involved in the singlet case; for low-order treatments see refs. [16]. This situation is far less severe for the contributions with quartic Casimir invariants,

$$
d_{x y}^{(4)} \equiv d_{x}^{a b c d} d_{y}^{a b c d}
$$

where $x, y$ labels the representations with generators $T_{r}^{a}$ and

$$
d_{r}^{a b c d}=\frac{1}{6} \operatorname{Tr}\left(T_{r}^{a} T_{r}^{b} T_{r}^{c} T_{r}^{d}+\text { five } b c d \text { permutations }\right),
$$

which occur in the splitting functions for the first time at four loops. This effective 'leading-order' situation implies particular relations and facilitates calculational simplifications. These include

$$
P_{\mathrm{qq}}^{(3)}(N)+P_{\mathrm{gq}}^{(3)}(N)-P_{\mathrm{qg}}^{(3)}(N)-P_{\mathrm{gg}}^{(3)}(N) \stackrel{\underline{\underline{ }}}{=} 0
$$

( $\underline{\underline{Q}}$ denotes equality for the quartic Casimir contributions) for the colour-factor substitutions [8]

$$
\left(2 n_{f}\right)^{2} d_{F F}^{(4)} / n_{a}=2 n_{f} d_{F A}^{(4)} / n_{a}=2 n_{f} d_{F F}^{(4)} / n_{c}=d_{F A}^{(4)} / n_{c}=d_{A A}^{(4)} / n_{a}
$$

that lead to an $\mathscr{N}=1$ supersymmetric theory; for lower-order discussions see refs. [17]. Moreover the off-diagonal quantities are found to be related by [10]

$$
P_{\mathrm{qg}}^{(0)}(N) P_{\mathrm{gq}}^{(3)}(N) \stackrel{Q}{=} P_{\mathrm{gq}}^{(0)}(N) P_{\mathrm{qg}}^{(3)}(N) .
$$

This second relation, which we have found empirically by inspecting our results, is consistent with the implications of $\mathscr{N}=1$ supersymmetry for QCD conformal operators investigated in ref. [18]. It is also a special case of the structure predicted in ref. [19] from the conformal symmetry of QCD at some non-integer space-time dimension $D=4-2 \varepsilon$.

We have used eqs. (3.4) and (3.5) partly to check the results of our diagrams calculations, and partly to simplify our computational task at the highest values of $N$. In this manner, we have been able to derive all $d_{x y}^{(4)}$ contributions to the $\mathrm{N}^{3} \mathrm{LO}$ splitting functions at $N \leq 16$. These results, and the structurally interesting all- $N$ expressions for the $\zeta_{5}$-terms, can be found in ref. [10].

Analogous to the non-singlet quantities analyzed in ref. [12], the moments of $P_{\mathrm{gg}}^{(3)}$ at $N \leq 16$ facilitate numerical determinations of the quartic-Casimir contributions to the four-loop gluon cusp anomalous dimension $A_{4, \mathrm{~g}}$, recall eq. (1.7). The present status of $A_{4, \mathrm{q}}$ and $A_{4, \mathrm{~g}}$ is collected in table 1 .

The coefficients of $A_{4, q}$ which are known exactly have also been determined from the quark form factor [20,21]; the results are in complete agreement. Recently, the exact coefficient of $C_{F}^{3} n_{f}$ has been obtained in ref. [22]. The only piece of $A_{4, \mathrm{~g}}$ known exactly so far is the $C_{A} n_{f}^{3}$ contribution [11,23]. For numerical results in $\mathscr{N}=4$ maximally supersymmetric Yang-Mills theory see ref. [24]. 


\begin{tabular}{cccc} 
quark & gluon & $A_{4, \mathrm{q}}$ & $A_{4, \mathrm{~g}}$ \\
\hline$C_{F}^{4}$ & - & 0 & - \\
$C_{F}^{3} C_{A}$ & - & 0 & - \\
$C_{F}^{2} C_{A}^{2}$ & - & 0 & - \\
$C_{F} C_{A}^{3}$ & $C_{A}^{4}$ & $610.25 \pm 0.1$ & \\
$d_{F A}^{(4)} / N_{F}$ & $d_{A A}^{(4)} / N_{A}$ & $-507.0 \pm 2.0$ & $-507.0 \pm 5.0$ \\
\hline$n_{f} C_{F}^{3}$ & $n_{f} C_{F}^{2} C_{A}$ & -31.00554 & \\
$n_{f} C_{F}^{2} C_{A}$ & $n_{f} C_{F} C_{A}^{2}$ & $38.75 \pm 0.2$ & \\
$n_{f} C_{F} C_{A}^{2}$ & $n_{f} C_{A}^{3}$ & $-440.65 \pm 0.2$ & \\
$n_{f} d_{F F}^{(4)} / N_{F}$ & $n_{f} d_{F A}^{(4)} / N_{A}$ & $-123.90 \pm 0.2$ & $-124.0 \pm 0.6$ \\
\hline$n_{f}^{2} C_{F}^{2}$ & $n_{f}^{2} C_{F} C_{A}$ & -21.31439 & \\
$n_{f}^{2} C_{F} C_{A}$ & $n_{f}^{2} C_{A}^{2}$ & 58.36737 & \\
- & $n_{f}^{2} d_{F F}^{(4)} / N_{A}$ & - & $0.0 \pm 0.1$ \\
$n_{f}^{3} C_{F}$ & $n_{f}^{3} C_{A}$ & 2.454258 & 2.454258 \\
\hline
\end{tabular}

Table 1: Fourth-order coefficients of the quark and gluon cusp anomalous dimensions determined from the large- $x$ limit $(1.7)$ of the quark-quark and gluon-gluon splitting functions. The errors in the quark case are correlated due to the exactly known large- $n_{c}$ limit. The numerical value of $-31.00 \pm 0.4$ of ref. [12] for the coefficient of $n_{f} C_{F}^{3}$ in $A_{4, q}$ has been replaced by the exact result of ref. [22]. This and the values for the $n_{f}^{2}$ and $n_{f}^{3}$ coefficients have been rounded to seven digits. Entries left blank for $A_{4, \mathrm{~g}}$ have not been calculated from diagrams so far, but are related to those for $A_{4, \mathrm{q}}$ by Casimir scaling. Entries marked by '-' do not exist.

As up to the third order [1], the corresponding quark and gluon entries in table 1 have the same coefficients (for now: as far as they have been computed, and within numerical errors). We refer to this (for now: conjectured) relation as generalized Casimir scaling.

Unlike for the lower-order coefficients, this relation does not have the consequence that the values of $A_{4, \mathrm{~g}}$ and $A_{4, \mathrm{q}}$ are related by a simple numerical Casimir scaling in QCD, i.e., a factor of $C_{A} / C_{F}=9 / 4$. However, this numerical Casimir scaling is restored in the large- $n_{c}$ limit of the quartic colour factors, and therefore also in the overall large- $n_{c}$ limit, see also ref. [25].

The results in table 1 and the generalized Casimir scaling lead to the following numerical results for the four-loop cusp anomalous dimensions in QCD, expanded in powers of $\alpha_{\mathrm{s}} /(4 \pi)$ :

$$
\begin{aligned}
& A_{4, \mathrm{q}}=20702(2)-5171.9(2) n_{f}+195.5772 n_{f}^{2}+3.272344 n_{f}^{3}, \\
& A_{4, \mathrm{~g}}=40880(30)-11714(2) n_{f}+440.0488 n_{f}^{2}+7.362774 n_{f}^{3},
\end{aligned}
$$

where the number(s) in brackets indicate the uncertainty of the preceding digit(s). Combining these results with the lower-order coefficients, one arrives at the very benign expansions

$$
\begin{aligned}
& A_{\mathrm{q}}\left(\alpha_{\mathrm{s}}, n_{f}=3\right)=0.42441 \alpha_{\mathrm{s}}\left[1+0.72657 \alpha_{\mathrm{s}}+0.73405 \alpha_{\mathrm{s}}^{2}+0.6647(2) \alpha_{\mathrm{s}}^{3}+\ldots\right], \\
& A_{\mathrm{q}}\left(\alpha_{\mathrm{s}}, n_{f}=4\right)=0.42441 \alpha_{\mathrm{s}}\left[1+0.63815 \alpha_{\mathrm{s}}+0.50998 \alpha_{\mathrm{s}}^{2}+0.3168(2) \alpha_{\mathrm{s}}^{3}+\ldots\right], \\
& A_{\mathrm{q}}\left(\alpha_{\mathrm{s}}, n_{f}=5\right)=0.42441 \alpha_{\mathrm{s}}\left[1+0.54973 \alpha_{\mathrm{s}}+0.28403 \alpha_{\mathrm{s}}^{2}+0.0133(3) \alpha_{\mathrm{s}}^{3}+\ldots\right]
\end{aligned}
$$


and

$$
\begin{aligned}
& A_{\mathrm{g}}\left(\alpha_{\mathrm{s}}, n_{f}=3\right)=0.95493 \alpha_{\mathrm{s}}\left[1+0.72657 \alpha_{\mathrm{s}}+0.73405 \alpha_{\mathrm{s}}^{2}+0.415(2) \alpha_{\mathrm{s}}^{3}+\ldots\right], \\
& A_{\mathrm{g}}\left(\alpha_{\mathrm{s}}, n_{f}=4\right)=0.95493 \alpha_{\mathrm{s}}\left[1+0.63815 \alpha_{\mathrm{s}}+0.50998 \alpha_{\mathrm{s}}^{2}+0.064(2) \alpha_{\mathrm{s}}^{3}+\ldots\right], \\
& A_{\mathrm{g}}\left(\alpha_{\mathrm{s}}, n_{f}=5\right)=0.95493 \alpha_{\mathrm{s}}\left[1+0.54973 \alpha_{\mathrm{s}}+0.28403 \alpha_{\mathrm{s}}^{2}-0.243(2) \alpha_{\mathrm{s}}^{3}+\ldots\right]
\end{aligned}
$$

The remaining uncertainties of the $\mathrm{N}^{3} \mathrm{LO}$ coefficients are practically irrelevant for phenomenological applications. Note that, due to the breaking of the numerical Casimir scaling especially in the $n_{f}^{0}$ parts of eqs. (3.6) and (3.7) and the cancellations between the terms without and with $n_{f}$, the numerical Casimir scaling is completely broken for the $\mathrm{N}^{3} \mathrm{LO}$ terms in eqs. (3.8) and (3.9).

\section{First results for the $\mathrm{N}^{4} \mathrm{LO}$ non-singlet splitting functions}

Using the recent implementation [26] of the local $\mathrm{R}^{*}$-operation [27], it is now possible, at least for the lowest values of $N$, to extend the FORCER calculations of the splitting functions to the $\mathrm{N}^{4} \mathrm{LO}$ contributions $P^{(4)}$ in eq. (1.3). The computational setup is similar to (but includes some efficiency improvements upon) that used for the beta function and Higgs decays at five loops in refs. [28, 29].

As a check specific to the present case, we have explicitly verified that $P_{\mathrm{ns}}^{(4)-}(N=1)$ vanishes in a calculation with one power of the gauge parameter. We have then calculated $P_{\mathrm{ns}}^{(4)+}(N=2)$ and $P_{\mathrm{ns}}^{(4)-}(N=3)$ for a general gauge group. The latter computation required an effort comparable to that for the $\mathrm{N}^{4} \mathrm{LO}$ corrections to $H \rightarrow g g$ in the heavy top-quark limit refs. [29], the hardest calculation performed so far with the program of ref. [26]. An extension to $N=4$ would be extremely hard with the present tools; higher values of $N$ are out of reach for now.

The analytic results will be presented elsewhere. Before turning to their numerical effects, it is worthwhile to mention another, if not particularly strong check: besides rational numbers,

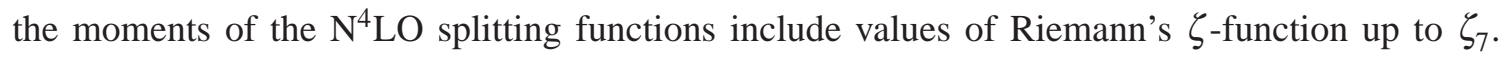
Consistent with the 'no- $\pi^{2}$ theorem' for Euclidean physical quantities [30], the $\zeta_{6}$ terms disappear when the $\overline{\mathrm{MS}}$ splitting functions are converted to physical evolution kernels for structure functions in DIS, and the $\zeta_{4}$ terms disappear after transforming to a renormalization scheme in which the $\mathrm{N}^{4} \mathrm{LO}$ beta function does not include $\zeta_{4}$-terms, such as MINIMOM in the Landau gauge $[31,32]$.

Our new results for $N=2$ and $N=3$ lead to the numerical $\overline{\mathrm{MS}}$ expansions

$$
\begin{aligned}
P_{\mathrm{ns}}^{+}(2,0) & =-0.2829 \alpha_{\mathrm{s}}\left(1+1.0187 \alpha_{\mathrm{s}}+1.5307 \alpha_{\mathrm{s}}^{2}+2.3617 \alpha_{\mathrm{s}}^{3}+4.520 \alpha_{\mathrm{s}}^{4}+\ldots\right), \\
& \ldots \\
P_{\mathrm{ns}}^{+}(2,3) & =-0.2829 \alpha_{\mathrm{s}}\left(1+0.8695 \alpha_{\mathrm{s}}+0.7980 \alpha_{\mathrm{s}}^{2}+0.9258 \alpha_{\mathrm{s}}^{3}+1.781 \alpha_{\mathrm{s}}^{4}+\ldots\right), \\
P_{\mathrm{ns}}^{+}(2,4) & =-0.2829 \alpha_{\mathrm{s}}\left(1+0.7987 \alpha_{\mathrm{s}}+0.5451 \alpha_{\mathrm{s}}^{2}+0.5215 \alpha_{\mathrm{s}}^{3}+1.223 \alpha_{\mathrm{s}}^{4}+\ldots\right), \\
P_{\mathrm{ns}}^{+}(2,5) & =-0.2829 \alpha_{\mathrm{s}}\left(1+0.7280 \alpha_{\mathrm{s}}+0.2877 \alpha_{\mathrm{s}}^{2}+0.1512 \alpha_{\mathrm{s}}^{3}+0.849 \alpha_{\mathrm{s}}^{4}+\ldots\right)
\end{aligned}
$$

and

$$
\begin{aligned}
P_{\mathrm{ns}}^{-}(3,0) & =-0.4421 \alpha_{\mathrm{s}}\left(1+1.0153 \alpha_{\mathrm{s}}+1.4190 \alpha_{\mathrm{s}}^{2}+2.0954 \alpha_{\mathrm{s}}^{3}+3.954 \alpha_{\mathrm{s}}^{4}+\ldots\right), \\
& \ldots \\
P_{\mathrm{ns}}^{-}(3,3) & =-0.4421 \alpha_{\mathrm{s}}\left(1+0.7952 \alpha_{\mathrm{s}}+0.7183 \alpha_{\mathrm{s}}^{2}+0.7605 \alpha_{\mathrm{s}}^{3}+1.508 \alpha_{\mathrm{s}}^{4}+\ldots\right), \\
P_{\mathrm{ns}}^{-}(3,4) & =-0.4421 \alpha_{\mathrm{s}}\left(1+0.7218 \alpha_{\mathrm{s}}+0.4767 \alpha_{\mathrm{s}}^{2}+0.3921 \alpha_{\mathrm{s}}^{3}+1.031 \alpha_{\mathrm{s}}^{4}+\ldots\right), \\
P_{\mathrm{ns}}^{-}(3,5) & =-0.4421 \alpha_{\mathrm{s}}\left(1+0.6484 \alpha_{\mathrm{s}}+0.2310 \alpha_{\mathrm{s}}^{2}+0.0645 \alpha_{\mathrm{s}}^{3}+0.727 \alpha_{\mathrm{s}}^{4}+\ldots\right) .
\end{aligned}
$$


Here we have included $n_{f}=0$ besides the physically relevant values, since it provides useful information about the behaviour of the perturbation series. The $\mathrm{N}^{4} \mathrm{LO}$ coefficients in (4.1) and (4.2) are larger than one may have expected from the NNLO and $\mathrm{N}^{3} \mathrm{LO}$ contributions.

It is interesting in this context to consider the effect of the quartic group invariants. For example, the $n_{f}=0$ coefficients in eqs. (4.1) and (4.2) at $\mathrm{N}^{3} \mathrm{LO}$ and $\mathrm{N}^{4} \mathrm{LO}$ can be decomposed as

$$
\begin{aligned}
& 2.3617=2.0878+0.1096 d_{F A}^{(4)} / n_{c} \\
& 4.520=3.552-0.0430 d_{F A}^{(4)} / n_{c}+0.0510 d_{A A}^{(4)} / n_{a}
\end{aligned}
$$

and

$$
\begin{aligned}
& 2.0954=2.0624+0.0132 d_{F A}^{(4)} / n_{c} \\
& 3.954=3.371-0.0171 d_{F A}^{(4)} / n_{c}+0.0371 d_{A A}^{(4)} / n_{a}
\end{aligned}
$$

with $d_{F A}^{(4)} / n_{c}=5 / 2$ and $d_{A A}^{(4)} / n_{a}=135 / 8$ in QCD, see, e.g., app. C of ref. [32]: Without the rather large contributions of $d_{A A}^{(4)}$, which enter at $\mathrm{N}^{4} \mathrm{LO}$ for the first time, the series would look much more benign with consecutive ratios of 1.4 to 1.6 between the $\mathrm{N}^{4} \mathrm{LO}, \mathrm{N}^{3} \mathrm{LO}$, NNLO and NLO coefficients. This sizeably $d_{A A}^{(4)}$ contribution $\left(\sim n_{c}^{2}+36\right)$ also implies that the leading large- $n_{c}$ contribution provides a less good approximation at $\mathrm{N}^{4} \mathrm{LO}$ than at the previous orders.

The numerical impact of the higher-order contributions to the splitting functions $P_{\mathrm{ns}}^{ \pm}$on the $N=2$ and $N=3$ moments of the respective PDFs (1.5) are illustrated in fig. 3. At $\alpha_{\mathrm{s}}\left(\mu_{f}^{2}\right)=0.2$ and $n_{f}=4$, the $\mathrm{N}^{4} \mathrm{LO}$ corrections are about $0.15 \%$ at $\mu_{r}=\mu_{f}$, roughly half the size of their $\mathrm{N}^{3} \mathrm{LO}$ counterparts. Varying $\mu_{r}$ up and down by a factor of 2 - the required additional terms for the splitting functions can be found to $\mathrm{N}^{4} \mathrm{LO}$, e.g., in eq. (2.9) of ref. [33] - one arrives at a band with a full width of about $0.7 \%$. The $\mathrm{N}^{3} \mathrm{LO}$ and $\mathrm{N}^{4} \mathrm{LO}$ corrections are about twice as large at a lower scale with $\alpha_{\mathrm{s}}\left(\mu_{f}^{2}\right)=0.25$ and $n_{f}=3$.
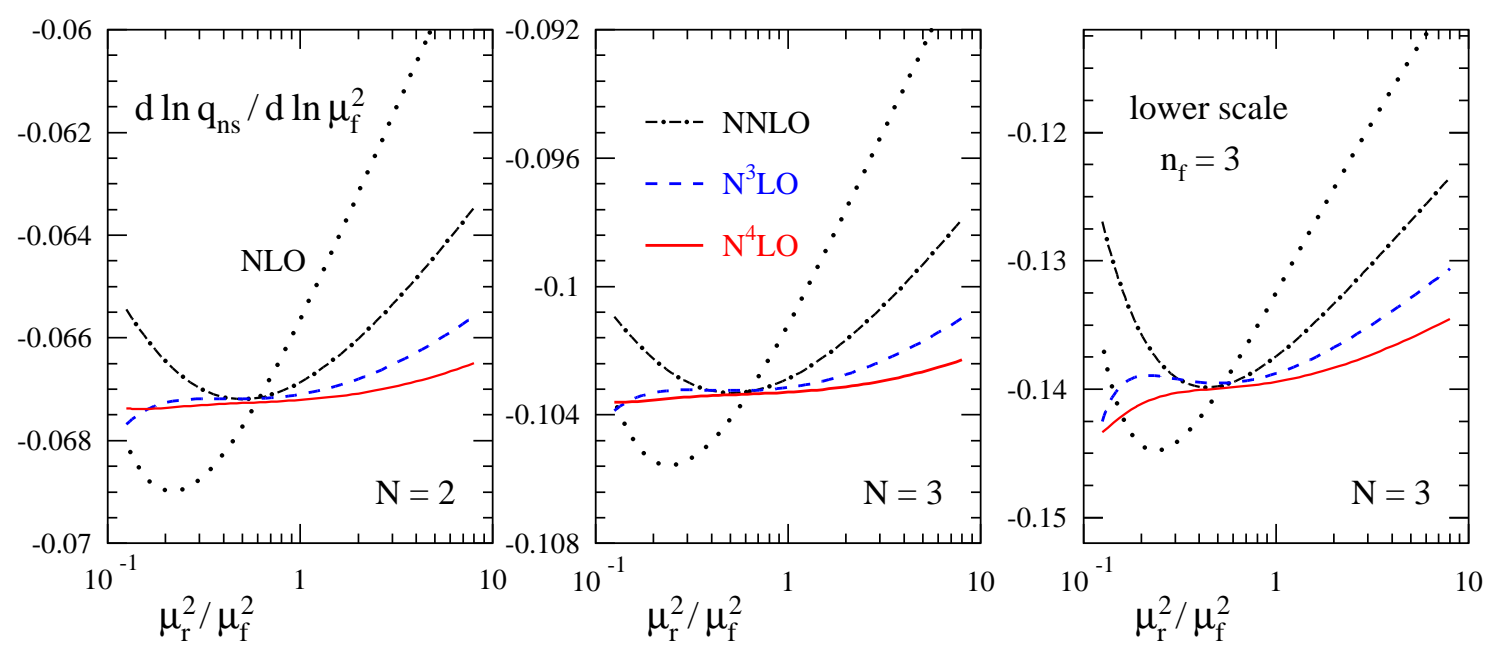

Figure 3: Left and middle panel: the renormalization-scale dependence of the logarithmic factorizationscale derivatives of the PDFs $q_{\mathrm{ns}}^{+}$at $N=2$ and $q_{\mathrm{ns}}^{-}$at $N=3$ at our standard reference point with $\alpha_{\mathrm{s}}\left(\mu_{f}^{2}\right)=0.2$ and $n_{f}=4$. Right panel: the corresponding $N=3$ results at a lower scale with $\alpha_{\mathrm{s}}\left(\mu_{f}^{2}\right)=0.25$ and $n_{f}=3$. 


\section{Acknowledgements}

A.V. is grateful to the organizers for support that facilitated his participation in this workshop. The research reported here has been supported by the Advanced Grant 320651, HEPGAME, of the European Research Council (ERC) and by the Deutsche Forschungsgemeinschaft (DFG) under grant number MO 1801/2-1.

\section{References}

[1] S. Moch, J.A.M. Vermaseren and A. Vogt, Nucl. Phys. B688 (2004) 101, hep-ph/0403192; A. Vogt, S. Moch and J.A.M. Vermaseren, Nucl. Phys. B691 (2004) 129, hep-ph/0404111

[2] S. Moch, J.A.M. Vermaseren and A. Vogt, Nucl. Phys. B889 (2014) 351, arXiv:1409.5131; S. Moch, J.A.M. Vermaseren and A. Vogt, Phys. Lett. B748 (2015) 432, arXiv:1506.04517

[3] J.A.M. Vermaseren, A. Vogt and S. Moch, Nucl. Phys. B724 (2005) 3, hep-ph/0504242; S. Moch, J.A.M. Vermaseren, A. Vogt, Nucl. Phys. B813 (2009) 220, arXiv:0812.4168; J. Davies, A. Vogt, S. Moch and J.A.M. Vermaseren, PoS (DIS 2016) 059, arXiv:1606.08907

[4] C. Anastasiou, C. Duhr, F. Dulat, F. Herzog and B. Mistlberger, Phys. Rev. Lett. 114 (2015) 212001, arXiv:1503.06056; C. Anastasiou et al., JHEP 05 (2016) 058, arXiv:1602.00695; B. Mistlberger, JHEP 05 (2018) 028, arXiv:1802.00833

[5] F.A. Dreyer and A. Karlberg, Phys. Rev. Lett. 117 (2016) 072001, arXiv:1606.00840

[6] J. Currie, T. Gehrmann, E.W.N. Glover, A. Huss, J. Niehues and A. Vogt, JHEP 05 (2018) 209, arXiv: 1803.09973

[7] P.A. Baikov, K.G. Chetyrkin and J.H. Kühn, Phys. Rev. Lett. 104 (2010) 132004, arXiv:1001.3606; P.A. Baikov, K.G. Chetyrkin, J.H. Kühn and J. Rittinger, Phys. Lett. B714 (2012) 62, arXiv:1206.1288

[8] B. Ruijl, T. Ueda, J.A.M. Vermaseren, J. Davies and A. Vogt, PoS (LL2016) 071, arXiv:1605.08408

[9] G.P. Korchemsky, Mod. Phys. Lett. A4 (1989) 1257

[10] S. Moch, B. Ruijl, T. Ueda, J. Vermaseren, A. Vogt, Phys. Lett. B782 (2018) 627, arXiv:1805.09638

[11] J. Davies, B. Ruijl, T. Ueda, J. Vermaseren, A. Vogt, Nucl. Phys. B915 (2017) 335, arXiv:1610.07477

[12] S. Moch, B. Ruijl, T. Ueda, J. Vermaseren and A. Vogt, JHEP 10 (2017) 041, arXiv:1707.08315

[13] A. Vogt, S. Moch, B. Ruijl, T. Ueda and J. Vermaseren, PoS (RADCOR 2017) 046, arXiv:1801.06085

[14] B. Ruijl, T. Ueda and J.A.M. Vermaseren, arXiv:1704.06650

[15] S.A. Larin, T. van Ritbergen and J.A.M. Vermaseren, Nucl. Phys. B427 (1994) 41; S. Larin, P. Nogueira, T. van Ritbergen, J. Vermaseren, Nucl. Phys. B492 (1997) 338, hep-ph/9605317

[16] R. Hamberg and W.L. van Neerven, Nucl. Phys. B379 (1992) 143; J.C. Collins and R.J. Scalise, Phys. Rev. D50 (1994) 4117, hep-ph/9403231; B.W. Harris and J. Smith, Phys. Rev. D51 (1995) 4550, hep-ph/9409405; Y. Matiounine, J. Smith and W.L. van Neerven, Phys. Rev. D57 (1998) 6701, hep-ph/9801224

[17] I. Antoniadis and E. G. Floratos, Nucl. Phys. B191 (1981) 217; A.A. Almasy, S. Moch and A. Vogt, Nucl. Phys. B854 (2012) 133, arXiv:1107.2263

[18] A.V. Belitsky, D. Müller and A. Schäfer, Phys. Lett. B450 (1999) 126, hep-ph/9811484 
[19] B. Basso and G. P. Korchemsky, Nucl. Phys. B775 (2007) 1, hep-th/0612247

[20] A. Grozin, J.M. Henn, G.P. Korchemsky and P. Marquard, JHEP 1601 (2016) 140, arXiv:1510.07803; A. Grozin, PoS (LL2016) 053, arXiv:1605.03886;

R.N. Lee, A.V. Smirnov, V.A. Smirnov and M. Steinhauser, Phys. Rev. D96 (2017) 014008, arXiv: 1705.06862

[21] J. Henn, A.V. Smirnov, V.A. Smirnov and M. Steinhauser, JHEP 1605 (2016) 066, arXiv:1604.03126; J. Henn, A.V. Smirnov, V.A. Smirnov, M. Steinhauser and R.N. Lee, JHEP 03 (2017) 139, arXiv:1612.04389

[22] A. Grozin, JHEP 06 (2018) 073, arXiv:1805.05050

[23] A. von Manteuffel and R. M. Schabinger, Phys. Rev. D95 (2017) 034030, arXiv:1611.00795

[24] R.H. Boels, T. Huber and G. Yang, Phys. Rev. Lett. 119 (2017) 201601, arXiv:1705.03444; R.H. Boels, T. Huber and G. Yang, JHEP 01 (2018) 153, arXiv:1711.08449

[25] L.J. Dixon, JHEP 01 (2018) 075, arXiv:1712.07274

[26] F. Herzog and B. Ruijl, JHEP 05 (2017) 037, arXiv:1703.03776;

B. Ruijl, F. Herzog, T. Ueda, J. Vermaseren, A. Vogt, PoS (RADCOR 2017) 011, arXiv:1801.06084

[27] K.G. Chetyrkin and F.V. Tkachov, Phys. Lett. B114 (1982) 340;

K.G. Chetyrkin and V.A. Smirnov, Phys. Lett. B144 (1984) 419;

K.G. Chetyrkin, Max-Plank-Institute preprint MPI-PH-PTH-13-91, available as arXiv:1701.08627

[28] F. Herzog, B. Ruijl, T. Ueda, J.A.M. Vermaseren and A. Vogt JHEP 02 (2017) 090, arXiv:1701.01404

[29] F. Herzog, B. Ruijl, T. Ueda, J.A.M. Vermaseren and A. Vogt, JHEP 08 (2017) 113, arXiv:1707.01044

[30] M. Jamin and R. Miravitllas, Phys. Lett. B779 (2018) 452, arXiv:1711.00787;

J. Davies and A. Vogt, Phys. Lett. B776 (2018) 189, arXiv:1711.05267;

P.A. Baikov and K.G. Chetyrkin, JHEP 06 (2018) 141, arXiv:1804.10088;

P.A. Baikov and K.G. Chetyrkin, arXiv:1808.00237 (these proceedings)

[31] L. von Smekal, K. Maltman and A. Sternbeck, Phys. Lett. B681 (2009) 336, arXiv:0903.1696

[32] B. Ruijl, T. Ueda, J.A.M. Vermaseren and A. Vogt, JHEP 06 (2017) 040, arXiv:1703.08532

[33] W.L. van Neerven and A. Vogt, Nucl. Phys. B603 (2001) 42, hep-ph/0103123 